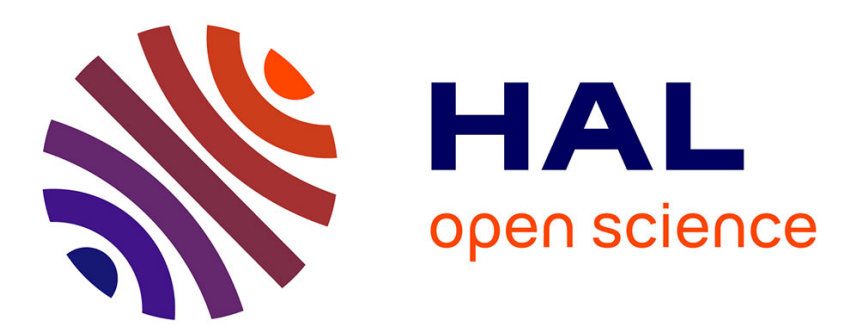

\title{
Pratiques des lieux, modes d'habiter, régimes d'habiter: pour une analyse trialogique des dimensions spatiales des sociétés humaines
}

Mathis Stock

\section{- To cite this version:}

Mathis Stock. Pratiques des lieux, modes d'habiter, régimes d'habiter: pour une analyse trialogique des dimensions spatiales des sociétés humaines. Travaux de l'Institut de Géographie de Reims, 2006, 115-118, pp.213-230. halshs-00716573

\section{HAL Id: halshs-00716573 \\ https://shs.hal.science/halshs-00716573}

Submitted on 10 Jul 2012

HAL is a multi-disciplinary open access archive for the deposit and dissemination of scientific research documents, whether they are published or not. The documents may come from teaching and research institutions in France or abroad, or from public or private research centers.
L'archive ouverte pluridisciplinaire HAL, est destinée au dépôt et à la diffusion de documents scientifiques de niveau recherche, publiés ou non, émanant des établissements d'enseignement et de recherche français ou étrangers, des laboratoires publics ou privés. 
Note de recherche

Pratiques des lieux, modes d'habiter, régimes d'habiter : pour une analyse trialogique des dimensions spatiales des sociétés humaines

Mathis Stock, EA HABITER (Université de Reims Champagne-Ardenne) \& EA MIT (Université de Paris 7) \& Laboratoire Chôros, Ecole Polytechnique Fédérale de Lausanne

Résumé

On a récemment ouvert un nouveau projet de questionnement géographique visant à explorer des manières dont les individus habitent les lieux géographiques du Monde (Stock, 2001 ; 2004). Le présent article propose trois notions interdépendantes qui pourraient nous aider à intégrer l'habiter des individus dans un contexte sociétal et spatial. Les termes de pratiques des lieux, modes/styles d'habiter et régimes d'habiter seront explorés quant à leur capacité heuristique à rendre compte des manières, changeantes, des sociétés à créer des référents géographiques pour et par leurs pratiques.

\section{mots-clés}

\section{Habiter - Individu - Mobilité géographique - Mode d'habiter - Pratiques - Modèles - Théorie - Régime d'habiter.}

\section{Abstract}

We recently proposed a new project for the geographical research based on the ways individuals inhabit the geographical places of the World (Stock, 2001; 2004). We now propose three interdependent concepts that could help us to embed the dwelling of the individuals into a societal and spatial context. The notions of "practice of places", "modes or styles of dwelling" and "regimes of dwelling" will be explored on behalf their heuristic capacity of grasping the changing manners, by societies, of creating geographical referents for and by practice.

\section{Keywords \\ Dwelling - Geographical mobility - Individual - models - mode of dwelling - Practice - regime of dwelling - theory.}

\section{Zusammenfassung}

Wir haben kürzlich ein neues Projekt für die geographischen Fragestellung vorgeschlagen, nämlich der Art und Weise, in der die Individuen die geographischen Orte der Welt bewohnen (Stock, 2001; 2004). Wir schlagen nun drei interdependente Konzepte vor, welche uns helfen könnten, die Individuen in einen sozietalen und räumlichen Kontext einzubeziehen. Die Begriffe der " Praxis der Orte ", der "Arten oder Style des Wohnens " und der "Regime des Wohnens" sollen hier auf ihre heuristische Fähigkeit, die sich wandelnden Arten und Weisen der Gesellschaften, geographische Fixierungen für und durch Praxis zu schaffen, erkundet werden.

Stichworte

Individuum - Geographische Mobilität - Modelle - Modus des Wohnens - Praxis Regime des Wohnens - Theorie - Wohnen.

\section{Introduction}

L'enjeu de la géographie réside, aujourd'hui plus que jamais, dans la construction d'une approche pertinente des dimensions spatiales des sociétés humaines contemporaines. Prendre en compte le contexte sociétal, travailler sur la société par de l'espace et par les spatialités, exige une recherche d'adéquation maximale des instruments 
cognitifs avec les caractéristiques des sociétés contemporaines ${ }^{1}$. Notamment, d'autres manières de faire avec les lieux géographiques y émergent, différentes d'autres types de sociétés. Ce point est fondamental : l'habiter change selon les sociétés, et la manière d'être et de faire avec les lieux géographiques ne sont pas les mêmes selon les civilisations et degrés de développement.

Celles-ci sont aujourd'hui fortement informées par la mobilité géographique et une "individualisation géographique" : d'où l'appellation " sociétés à individus mobiles " que l'on peut leur assigner d'un point de vue géographique (Stock, 2001). On peut penser que l'accroissement de la mobilité ainsi que la différenciation des rythmes de déplacements ont pour conséquence un changement de signification des différents lieux géographiques que les individus pratiquent.

On assiste ainsi à une recomposition des valeurs assignées aux lieux qui change radicalement la manière dont les individus habitent les lieux. C'est en ces termes que se pose dorénavant la question de la géographicité des hommes/femmes vivant dans les sociétés à individus mobiles. Comment les individus habitent-ils les lieux géographiques du Monde? Voilà la question fondamentale qui exprime la dimension géographique de l'être humain (Mensch) et dont les qualités changeantes au cours du temps requièrent de prendre une distance critique par rapport aux manières habituelles de faire/penser/écrire la géographie.

En ce sens, nous posons que cette mobilité géographique accrue - donc la multiplication des lieux pratiqués par les individus ainsi que les circulations effectuées à travers des itinéraires - définit et exprime de nouvelles pratiques des lieux, de nouveaux modes d'habiter et un nouveau régime d'habiter. Le fait de pratiquer une multiplicité de lieux aurait pour conséquence, - c'est l'hypothèse -, l'émergence d'un nouveau rapport aux lieux géographiques, aux agencements spatiaux, à la distance et au mouvement, mais aussi, par ricochet, à Soi et à l'Autre. Ainsi, habiter, défini uniquement à l'échelle des pratiques des lieux des individus (Stock, 2001 ; 2004) ou comme spatialité individuelle (Lévy \& Lussault, 2003), néglige deux dimensions fondamentales : l'interdépendance des pratiques et la socialisation ainsi que, plus généralement, l'inscription de l'individu dans une société et un Monde qui le dépasse, mais l'inclut. Afin de prendre en compte ces deux dimensions, je propose les notions de mode d'habiter et de régime d'habiter qui sont articulées aux pratiques des lieux.

Dans cet article, quatre aspects sont travaillés : d'abord, la pertinence de la question de l'habiter éloigne la problématique de la Terre pour se centrer sur celle des lieux géographiques. Ensuite, la question des pratiques constitue la perspective centrale, du point de vue des individus, de la démarche. Le troisième point concerne les modes d'habiter ou styles d'habiter qui délaissent les pratiques prises isolément pour une vue d'ensemble. Enfin, la notion de régime d'habiter synthétise, au niveau sociétal, un ordre et un ordonnancement des rapports à de l'espace.

\section{Le questionnement des sociétés par l'habiter}

La question de l'habiter permet d'interroger d'une nouvelle façon, c'est-à-dire en mettant au centre les pratiques des individus, les dimensions spatiales des sociétés contemporaines. L'habiter est un terme qui a surtout été utilisé dans la géographie phénoménologique du rapport entre l'homme et la Terre, notamment d'inspiration heideggerienne (cf. Seamon \& Mugerauer, 1989). Pour Heidegger ([1952], 2004a), habiter signifie un " trait fondamental de l'être-là humain" (p. 181) : “Être humain signifie : être sur la Terre en tant que mortel, c'est-àdire : habiter" ([1952], 2004b, p. 141) ${ }^{2}$. Cet être-sur-la-Terre, cet habiter, y a deux expressions : 1) habiter au sens d'avoir des habitudes dans le quotidien, 2) bâtir qui signifie une autre modalité d'être en

\footnotetext{
${ }^{1}$ Je tente ici d'éviter l'article "le " ou " un " lorsque j'utilise le terme "espace ". Cette tentative s'inscrit dans la voie ouverte par Benno Werlen $(1995$; 1997) qui indique que " espace " peut être utilisé comme concept classificatoire, mais ne devant pas devenir lui-même une partie de la classe "espace". Ainsi, les expressions "l'espace " ou "un espace " sont remplacées par les expressions " de l'espace" et "d'espace" (production ou constitution d'espace par exemple). On trouve dans le texte Khôra de Jacques Derrida (1993) le même problème concernant l'interprétation du texte Timaios de Platon où la question de «chôra », donc du lieu ou de l'espace est posée. Pour ce problème de sens et de traduction du terme « chôra » par rapport à « topos », Berque (2005) a proposé la solution suivante : " milieu existentiel » par rapport à « lieu ». On pourrait également proposer « lieu » (Ort) par rapport à « endroit» (Stelle). Cf. Bollnow (1963) pour une analyse approfondie du sens des termes Ort, Raum, Platz, Stelle.

${ }^{2}$ Cependant, la géographie scientifique à partir du $19^{\text {ème }}$ siècle propose de considérer la Terre comme la «demeure » de l'homme, position prolongée dans la géographie française par la notion d' «homme-habitant» de Maurice Le Lannou (1949) : "Habiter, c'est vivre sur un morceau de la planète, en (sic) tirer de quoi satisfaire les besoins élémentaires (sic) de l'existence, et dans une mesure variable, un certain nombre de besoins (sic) acquis et de commodités superflues (sic) ". Ainsi, "la géographie humaine est la science de l'homme-habitant" (1949). Ces formulations sont aujourd'hui dépassées et ne méritent leur mention qu'au titre d'exhaustivité historique : 1) vision sédentaire et agraire ; 2) vision comportementale (les
} 
rapport avec la Terre : “enclore”, "soigner", “cultiver". Hoyaux $(2000 ; 2001 ; 2002 ; 2003)$ utilise cette conception heideggerienne et définit l'habiter comme la constitution de l'individu par les lieux géographiques. Cette perspective n'est pas adoptée ici, et ce pour différentes raisons : le survalorisation du Sujet au détriment du social, la perspective rurale où la proximité et le local ont une valeur positive, toujours postulée, jamais démontrée, le rejet du lointain et de l'ailleurs dans la sphère du dangereux etc ${ }^{4}$. Pour résumer, " habiter » n'est pas non plus utilisé dans le sens d'une pratique " habituelle" ou «familière » de l'espace, une utilisation du lieu de résidence comme c'est encore trop souvent le cas (cf. Mayol, 1990 ; Chalas, 2000) ni comme un certain rapport à la Terre.

La perspective adoptée est celle des pratiques des lieux des individus (Stock, 2001), permettant ainsi d'intégrer des pratiques et des lieux non-habituels, du «hors-quotidien », de l'éphémère. Selon Lévy \& Lussault (2003), habiter désigne "la spatialité des acteurs individuels" (p. 440). Il permet de se situer du côté de la spatialité et non pas de l'espace, deux catégories distinctes selon Lussault (2003), c'est-à-dire de ce qui relève des actions spatiales des individus à travers différentes technologies et instruments ainsi que leurs langages et imaginaires. On peut synthétiser ces propositions en affirmant que l'habiter est défini comme étant le rapport à de l'espace tel qu'il est exprimé par les pratiques humaines, ce qui est proche de la "Praxis der Weltbindung », conceptualisée par Werlen $(1996 ; 1997)$.

Il s'agit d'un projet cognitif qui prend au sérieux l'individu comme acteur géographique et comme question centrale pour la géographie. Travailler sur l'individu d'un point de vue géographique est l'une des avancées scientifiques récentes; aborder l'habiter comme étant centré sur les pratiques des lieux des individus tente de donner une cohérence à ce projet scientifique qui reste théoriquement à concrétiser ${ }^{5}$. Ce programme scientifique peut être compris, épistémologiquement, comme étant un positionnement qui permet une reconnaissance de la plus grande complexité des objets humains, notamment en raison de la capacité symbolique des membres des sociétés humaines, par rapport aux objets des sciences physico-chimiques qui sont les moins complexes, et les objets des sciences du vivant, moyennement complexes (Elias, 1970). De ce point de vue, le projet de l'habiter n'est pas réductible au courant dit de l'analyse spatiale qui correspond à une tentative de "physique sociale" c'est-à-dire qui fait comme si les objets humains avaient le même degré de complexité que les objets des sciences physico-chimiques - ni au courant de la géographie sociale comportementale (cf. Golledge \& Stimson, 1997) - où les objets humains sont réduits aux entités physiologiques qui réagissent aux stimuli de l'environnement à travers les perceptions. Ces approches pèchent par un réductionnisme dans leurs tentatives d'explication : elles simplifient excessivement, voire évacuent les intentionnalités et stratégies humaines ${ }^{6}$.

Dans le même temps, il convient de ne pas isoler l'individu, ne pas faire comme si l'individu était un " homo clausus", comme l'appelle Norbert Elias (1970), mais relié, en interdépendance avec d'autres individus ainsi qu'avec les normes et valeurs sociales et inséré dans différents contextes d'action. De ce point de vue, il convient d'aller plus loin que les analyses centrées sur le sujet en géographie depuis les années 1970. Ainsi, "1'habiter" ne peut se ramener seulement à l'individu, mais demande à être analysé d'un triple point de vue articulé : les pratiques des individus, l'ensemble des pratiques des individus, et des éléments ordonnés qui dépassent l'individu mais qui font partie de son habiter. D'où l'exploration de trois notions interdépendantes : pratiques des lieux, modes/style d'habiter et régime d'habiter qui correspondent à trois niveaux d'analyse de l'habiter.

\footnotetext{
" besoins élémentaires" définies une fois pour toutes ; 3) "commodités superflues" qui ne sont justement superflues que dans une vision de survie physique alors même qu'elles assurent le maintien de Soi et l'acceptation dans un communauté ou un groupe.

${ }^{3}$ Il y ajoute une troisième idée : c'est cet habiter qui, à travers le bâtir, mène à la création de lieu $($ Ort $)$ et d'espace $($ Raum $)$ sur une Terre pourvue d'emplacements (Stelle). C'est ce que Henri Lefebvre prolonge : "L'habiter s'exprime objectivement dans un ensemble d'œuvres, de produits, de choses qui constituent un système partiel : la maison, la ville ou l'agglomération " (Lefebvre, 1999, p. 166). Ainsi se trouve exprimée la distinction que font Lévy \& Lussault (2003) de l'habitat comme organisation matérielle et de l'habiter comme spatialité de l'acteur.

${ }^{4}$ Il conviendrait de développer ici une critique rigoureuse de la conception de l'habiter et de l'espace de Heidegger, centrée sur le proche comme assurant la « sécurité ontologique». Cf. Stock (2004) pour la justification d'une autre perspective de l'habiter.

${ }^{5}$ On peut faire remonter l'attention aux acteurs en géographie aux années 1980 (cf. Werlen, 1987), au Sujet aux années 1970 (Relph, 1971 ; Tuan, 1971 ; Frémont, 1972).

${ }^{6}$ Cela étant dit, il est nécessaire de travailler sérieusement sur les articulations entre ces trois différents styles épistémologiques, d'en souligner les apports et les limites d'un point de vue cognitif. Peut-on les articuler les uns aux autres ou bien forment-elles des points de vue incommensurables? Ce sont ces questions qu'il convient de poser et auxquelles il sera nécessaire de répondre. D'ores et déjà, on peut oser l'hypothèse que les procédures de types « analyse spatiale » arrivent à une certaine mise en ordre descriptive et une qualification du monde des sociétés humaines, mais que l'enchaînement explicatif digne des standards scientifiques en vigueur dans les sciences humaines et sociales résiste à ces modèles.
} 


\section{Les pratiques des lieux font partie de l'habiter}

Appréhender l'habiter par les pratiques des individus ne va pas de soi, mais semble un parti pris indispensable pour connaître, avec plus de précision que ne le font les modèles classiques, les rapports à de l'espace. On peut énoncer les arguments suivants pour ce choix :

- les lieux et l'habiter ne font sens qu'en situation, en acte (Lussault, 2000), dans des " situations problématiques" (Foucault, 1984, Popper, 1998, Werlen, 1997) ; selon les situations, les sens assignés aux lieux géographiques varient (Stock, 2001).

- on observe une dissociation de plus en plus forte entre emplacement des pratiques humaines et lieu de résidence, processus que la recherche en géographie a du mal à saisir: l'approche par les pratiques conduit à analyser plus finement les associations pratiques/lieux des individus.

- les représentations des lieux géographiques et de l'agencement spatial s'établissent en fonction des pratiques, en fonction d'un projet de l'individu ; notamment, la mobilité géographique contribue à former des compétences spatiales qui constituent l'imaginaire géographique.

- la " pluralité " des ressorts de l'action (Lahire, 1998) font qu'il existe une "variation intra-individuelle " - en fonction de différentes situations - et non seulement une "variation inter-société " ou "interindividuelle" (Lahire, 2004) : il s'agit donc d'une précision par rapport à d'autres approches. Le sens accordé aux lieux géographiques varie notamment, pour un même individu, selon le projet pratique à travers lequel il est abordé (Stock, 2001).

Comment définir les pratiques des lieux ? Pour Lefebvre (1999), la pratique spatiale " englobe production et reproduction, lieux spécifiés et ensembles spatiaux propres à chaque formation sociale, qui assure la continuité dans une certaine cohésion. Cette cohésion implique pour ce qui concerne l'espace social et le rapport à son espace de chaque membre de telle société, à la fois une compétence certaine et une performance" (p. 42, souligné dans l'original). Lefebvre (1999 : 70) défend la thèse selon laquelle l'espace est " posé avant la venue de l'acteur" ; les pratiques s'effectuent ainsi dans de l'espace toujours-déjà-là, ce qui est à la fois vrai et pose en même temps un problème. Étant donné que l'acteur contribue également à constituer de l'espace, voire à le créer, à le faire advenir - par exemple la venue des touristes pendant la "saison" transforme un lieu à infrastructures touristiques en un "véritable" lieu touristique par la présence des touristes -, la formulation induit en erreur et interdit d'appréhender les lieux et agencements comme étant de l'espace habité.

Pour effectuer ce tournant, on peut s'appuyer sur les travaux de Michel de Certeau (1990) et définir les pratiques spatiales comme étant des manières de faire avec de l'espace. Plus précisément, Michel de Certeau (1990) nomme " pratique du lieu" le fait de déployer les pratiques pour que le lieu devienne espace :

\footnotetext{
“Est un lieu l'ordre (quel qu'il soit) selon lequel des éléments sont distribués dans des rapports de coexistence. S'y trouve donc exclue la possibilité, pour deux choses, d'être à la même place. La loi du 'propre' y règne : les éléments considérés sont les uns à côté des autres, chacun situé en un endroit 'propre' et distinct qu'il définit. Un lieu est donc une configuration instantanée de positions. Il implique une indication de stabilité. Il y a espace dès qu'on prend en considération des vecteurs de direction, des quantités de vitesse et la variable du temps. L'espace est un croisement de mobiles. Il est en quelque sorte animé par l'ensemble des mouvements qui s'y déploient. Est espace l'effet produit par les opérations qui l'orientent, le circonstancient, le temporalisent et l'amènent à fonctionner en unité polyvalente de programmes conflictuels ou de proximités contractuelles. L'espace serait au lieu ce que devient le mot quand il est parlé, c'est-à-dire quand il est saisi dans l'ambiguité d'une effectuation, mué en un terme relevant de multiples conventions, posé comme l'acte d'un présent (ou d'un temps), et modifié par les transformations dues à des voisinages successifs. À la différence du lieu, il n'a donc ni l'univocité ni la stabilité d'un 'propre'. En somme l'espace est un lieu pratiqué" (p.173, souligné dans l'original).
}

Issue de cette réflexion, la notion "pratiques des lieux" sert à exprimer que ce n'est pas une pratique qui s'oppose à l'espace mais qui fait avec de l'espace. En fait, elle fait plus avec les lieux, car, comme le dit Michel de Certeau, c'est à travers la pratique que le lieu est transformé en espace. L'espace ne préexiste donc pas à la pratique, mais n'émerge qu'à la faveur des pratiques. L'idée est que chaque pratique exprime un certain habiter $\grave{a}$ l'échelle de l'individu, un certain rapport à l'ici et l'ailleurs, au quotidien et au hors-quotidien, aux lieux 
familiers, associée à un déplacement ou non, à la qualité du lieu et aux accessibilités, à la mobilité et aux métriques. C'est cette idée qui sous-tend, par exemple, l'analyse des pratiques touristiques comme une manière d'habiter les lieux touristiques par les touristes : par un déplacement pour une pratique de recréation temporaire in situ, de façon distanciée, ce qui implique une certaine altérité à gérer ${ }^{7}$.

Dans les sociétés à individus mobiles, les associations pratiques/lieux géographiques se posent avec de plus en plus d'acuité - deviennent " problématiques" au sens de Foucault (1984) : il faut décider de règles énonciatives - , dans le sens où les individus doivent faire avec des lieux de plus en plus nombreux. Ceci est dû à la mobilité géographique accrue qui exprime le fait que les lieux géographiques associés aux pratiques sont de plus en plus nombreux et de plus en plus éloignés les uns par rapport aux autres, relativement à d'autres " régimes d'habiter". Tentons de lister quelques pratiques qui aujourd'hui sont associées à des lieux plus ou moins éloignés du ou des lieux de domicile, et ce sans souci d'exhaustivité ${ }^{8}$ : mouvements "pendulaires ", voyages d'affaires, séminaire d'intégration, incentive, travailler comme VRP, aller à un congrès ${ }^{9}$; aller au cinéma, partir en vacances, visiter une ville, se rendre dans une "base régionale de loisir", aller dans sa maison de campagne ou celle d'amis, faire un "spring break" en tant qu'étudiant états-unien ou canadien ou prendre le Loveboat pour les étudiants finlandais entre Turku et Stockholm; apprendre l'anglais à Malte ou à Brighton, apprendre le français à Paris, en fait des pratiques qui associent un lieu autre à une pratique relativement plus contraignante que les loisirs ; faire le plein d'essence en Autriche ou au Luxembourg pour les Allemands, acheter des cigarettes au Luxembourg pour les Lorrains, exploitant ainsi un différentiel de prix ; faire des achats pendant son voyage de vacances ; se faire soigner dans un hôpital français ou espagnol pour les Anglais, se faire avorter en Belgique ou en Espagne ; faire du shopping - les soldes de Londres pour les Européens et Israéliens -, faire les courses dans les usines center, dans un centre commercial, en centre-ville, mais aussi, pour les frontaliers, dans un autre pays : les Anglais dans le Nord-Pas-de-Calais, les Suisses dans le Jura et en Savoie, les Allemands à Strasbourg, les Strasbourgeois à Kehl, etc ; aller voir ses amis et sa famille afin de garder un lien d'amitié etc. ${ }^{10}$.

Il s'agit là de pratiques des lieux in situ, avec un déplacement à la clé, plus précisément une circulation (cf. Stock $\&$ Duhamel, 2005) ${ }^{11}$. Toutefois, toutes les pratiques se ne valent pas, mais se différencient notamment en fonction de l'accessibilité et du sens, de l'intentionnalité de la pratique : certaines se déroulent dans le quotidien, d'autres dans le hors-quotidien" ${ }^{12}$. Ainsi, chaque pratique exprime l'habiter par un certain " rapport à de l'espace": qualités des lieux associées à la pratique, sens accordés aux lieux, propre à chaque pratique. Par exemple, les pratiques touristiques se caractérisent par un habiter temporaire où les pratiques de recréation, c'està-dire le relâchement de l'auto-contention par rapport au quotidien par les trois modalités découvrir, jouer, se reposer, sont associées à un lieu autre, investi par l'individu comme étant relativement plus distancié que le lieu du quotidien (Knafou et al., 1997 ; Stock, 2001, Sacareau \& Stock, 2003 ; Stock, 2005).

Fondamentalement, nous faisons l'hypothèse que les pratiques intègrent chacune une certaine qualité intrinsèque, avec une autonomie et des degrés de liberté plus ou moins grands quant au problème d'espace. Ainsi, les dimensions spatiales de l'individu ont radicalement changé. Il s'agit d'individus dont les référents géographiques de l'identité, des valeurs, des pratiques sont relativement plus autonomes, avec davantage de choix, de ce qu'ils étaient dans une société “ industrielle ”.

\footnotetext{
${ }^{7}$ Cf. Urry (1990), Rémy (1996), Hennig (1997), Knafou et al. (1997), Stock (2001; 2005), Équipe MIT (2002), Ceriani et al. $(2004 ; 2005)$ pour la mise en place de cette idée.

${ }^{8}$ Il s'agit de pratiques collectées non pas à travers une enquête scientifique, mais à travers de lectures de différents médias, surtout des journaux.

${ }^{9}$ Il s'agit de pratiques qui s'effectuent dans la sphère du travail où un quart des déplacements se passe en dehors du lieu de travail habituel (Crague, 2003). Par ailleurs, l'enquête "Transports" de l'INSEE connaît les "motifs" de déplacements suivants : motif " lieu de travail fixe et habituel ", motif " lieu de travail non fixe : chantier, contacts ou réunions, visites à des clients, fournisseurs, sous-traitants, tournée professionnelle, VRP, repas d'affaires " ; motif "stage, conférence, congrès, formations, exposition professionnelle (dans un lieu différent du lieu de travail habituel) "; " autres motifs professionnels non désignés par ailleurs " (Crague, 2003, p. 195).

${ }^{10}$ Toutes ces pratiques nécessiteraient une analyse scientifique approfondie quant à la valeur assignée aux lieux par les individus, les manières de les pratiquer ainsi que les compétences spatiales mobilisées pour les effectuer. Ce programme ne pourra être réalisé ici.

${ }^{11}$ Dans le même temps, les circulations sont liées aux télé-communications : téléphone, courrier électronique ou lettre nous dispense certes d'une rencontre face-à-face, mais prépare souvent une circulation. Des exemples abondent : se faire livrer une pizza, préparer un voyage ou un rendez-vous professionnel etc.

${ }^{12}$ Cf. Knafou et al. (1997), Stock (2001), Sacareau \& Stock (2003), Stock \& Duhamel (2005), Stock (2005) pour la distinction quotidien/hors-quotidien qui se fonde précisément sur des critères géographiques d'accessibilité et d'altérité/étrangeté des lieux. Une difficulté réside toutefois dans le fait que tantôt on décrit une pratique, tantôt la manière dont on se déplace. La notion "pratique des lieux" tente d'englober les deux aspects.
} 


\section{Modes d'habiter, styles d'habiter}

L'analyse des pratiques des lieux ne peut être conduite de façon isolée, elle doit prendre en compte virtuellement toutes les pratiques des individus. Là réside, intrinsèquement, la faiblesse des études sur les pratiques urbaines et les choix résidentiels, dont la focalisation sur une seule pratique fait perdre de vue les liens avec d'autres pratiques ${ }^{13}$. Il convient donc de commencer à les articuler les unes aux autres. La notion de "système de mobilité " (Bassand et al., 1985 ; Kaufmann et al., 1997 ; Knafou, 1998) peut être utilisée afin d'y parvenir, en la transposant du niveau sociétal au niveau individuel par la conception de "systèmes individuels de mobilité" (Stock, 2001) dans lesquels sont agencées, au cours du temps, circulations et migrations. Ainsi, ce sont les manières spécifiques des individus d'articuler les pratiques des lieux qui définissent les " modes d'habiter". Par hypothèse, ces modes d'habiter existent dans un continuum entre " mono-topique" et "poly-topique" (cf. fig. $1)$.

La notion de mode d'habiter est classiquement utilisée afin de mettre en avant la manière dont les individus résident et le rapport à l'espace que ce mode résidentiel (HLM, pavillon, appartement bourgeois, péri-urbain etc.) exprime. Cette optique résidentielle a conduit à des études approfondies sur le pavillonnaire ou les grands ensembles (cf. Haumont et al., 1999). La question de l'habiter a également incité Mayol (1990) à proposer une étude sur la vie des individus dans le quartier lyonnais de la Croix-Rousse. Toutefois, restreindre l'habiter à la seule sphère résidentielle conduit à sous-estimer les autres lieux pratiqués. Comme l'avait déjà souligné Piolle $(1990 ; 1991)$, on assiste à une territorialisation multiple des individus, ce que la notion "société d'archipels" de Viard (1994) rend moins adéquatement, car il désigne les lieux, mais non les liens entre les lieux. Ceci permet d'envisager une extension de ce qu'on appelle "l'espace de vie ", c'est-à-dire l'ensemble des lieux pratiqués, non seulement ceux du quotidien ${ }^{14}$.

Les modes d'habiter peuvent donc être définis comme étant l'ensemble des pratiques des lieux des individus. C'est d'ailleurs de cette façon que l'on peut articuler la dimension spatiale et la dimension sociale : les modes d'habiter, par hypothèse, expriment des styles de vie ${ }^{15}$. On dispose de deux contributions à cette problématique : celle de l'Erlebnisgesellschaft (Schulze, 1997) qui produit des styles de vie fondés sur le ludique, l'expressif et l'esthétique, incluant ce qui permet de faire l'expérience de sensations fortes ${ }^{16}$; celle d'ancrages à des niveaux d'échelle différents selon une maîtrise différentielle des métriques (Lévy \& Haegel, 1997).

Afin de comprendre le changement des modes d'habiter, on peut tenter une modélisation de l'ensemble des pratiques des lieux, en distinguant deux modes d'habiter à l'aide de lieux plus ou moins familiers pour les individus ( $c f$. figure 1). Les points foncés indiquent les lieux familiers, les points en clair les lieux non familiers : ce modèle graphique signifie que, contrairement à ce qui se passe pour d'autres sociétés ou à d'autres époques, les lieux proches ne sont plus nécessairement ceux qui sont les mieux connus et les plus familiers. On voit notamment dans ce modèle que les lieux familiers peuvent être situés à des distances plus grandes que le rayon marquant la limite de l'espace de proximité. La variable discriminante pour déterminer la familiarité d'avec les lieux n'est plus la distance, mais la fréquence de la pratique. Le second cercle symbolise l'accroissement de l'accessibilité à partir d'un lieu - supposé en forme de cercles concentriques conformément aux modèles spatiaux présupposant l'isotropie - mais dont la conceptualisation ne rend pas compte des accessibilités

\footnotetext{
${ }^{13}$ Traditionnellement, les choix résidentiels sont analysés en fonction du lieu de travail bien que Lefebvre (1999) entrevoit aussi la détermination du lieu de résidence par les lieux de loisir.

${ }^{14}$ Cette notion, définie comme étant l'espace habituellement pratiqué (cf. Frémont et al., 1984), est appelée à être dépassée ou amendée : en effet, on élude les lieux pratiqués en dehors des habitudes, mais pour quelle raison? Les pratiques touristiques constituent un exemple frappant : elles sont entrées dans les mœurs, mais ne s'effectuent pas (toujours) dans les mêmes lieux bien qu'il y ait des pratiques de recréation dont la récurrence du choix des lieux soit constitutive de leur spécificité. Cet exemple sert à montrer les limites de la notion et une possibilité d'aller au-delà : il suffit de définir comme espace de vie, l'ensemble des lieux pratiqués au quotidien et dans le hors-quotidien, peu importe la durée ou la récursivité des pratiques des lieux. Cf. Stock (2001) pour une analyse détaillée de la notion " espace de vie ".

${ }^{15}$ D'où la possibilité, peut-être, de les nommer "styles d'habiter".

${ }^{16}$ Le terme allemand "erleben" est difficile à traduire en français, car il comporte d'autres connotations que "vivre", notamment "faire l'épreuve », "faire l'expérience» (Cassin, 2004). En phénoménologie, on fait la distinction entre "erleben" et gestalten", entre "vivre" et "concevoir", sans doute exploité par Lefebvre (1974) pour la distinction entre “espace vécu” et "espace conçu”. Dans le cas de Schulze (1997), cela signifie plutôt une manière particulière d'appréhender le vécu, c'est-à-dire par l'attention donnée à l'expressivité des pratiques. Plus précisément, une attention plus grande à la composante du plaisir, de l'esthétique et du ludique dans les pratiques et la production des biens de consommation et des services.
} 
différentielles ; il pourrait donc être élaboré avec plus de précision par un modèle centré sur les accessibilités localement meilleures.

Figure 1: Les modèles du style d'habiter mono-topique et du style d'habiter poly-topique

Source : Stock (2001)

Il s'agit là d'un modèle grossier qui est invalidé par les pratiques de double résidence car il fait comme s'il n'y avait qu'un seul lieu de résidence. Il pose également problème parce que la conception d'espace isotrope distances égales dans toutes les directions - n'est pas la meilleure approximation à la réalité. Enfin, des modèles plus précis existent, allouant un certain budget de temps à chacun des lieux pratiqués (cf. Lévy, 2004). L'unique objectif de ce modèle réside dans la contribution à la résolution d'un problème : faire comprendre que ce ne sont pas les distances en cercles concentriques qui régissent l'espace de vie et la familiarité des lieux - comme cela est stipulé par le modèle des " coquilles du moi" de Moles (1995) -, mais la mobilité géographique ${ }^{17}$. On aboutit ainsi, en travaillant empiriquement, au classement de lieux plus ou moins familiers qui permettent de distinguer différents modes ou styles d'habiter.

\section{Régimes d'habiter}

Pratiques des lieux et mode d'habiter comme manières individuelles d'habiter le Monde constituent un "regard"/"théorie" - theoreia signifie contemplation - qui laisse de côté le jeu des institutions, normes, infrastructures, valeurs sociales etc. dans lequel les premiers s'insèrent. La notion de "régime d'habiter" tente de les y intégrer, en utilisant l'idée d'assemblage ordonné et ordonnant de plusieurs éléments en interaction que rend également de terme de système : on pourrait aussi parler de systèmes d'habiter.

On peut retracer, entre autres, les utilisations suivantes du terme "régime". Il est utilisé par le courant économique de l'école de la régulation afin de décrire et expliquer les changements du capitalisme. Ils définissent comme "régime d'accumulation" un ensemble de pratiques et de normes de production et de consommation. Puis, la notion d" urban regime" de la théorie urbaine nord-américaine tente, dans une perspective institutionnelle, de rendre compte du développement urbain depuis 1970 (Soja, 2001). Un régime urbain est défini comme un "agencement de personnes de pouvoir en un groupe" qui vise à gérer l'espace urbain (Soja, 2001). Enfin, François Hartog (2003) définit des "régimes d'historicité " comme une mise en ordre du temps ou, mieux, des temporalités ${ }^{18}$. Cette notion est destinée à décrire et expliquer les rapports différents que les hommes construisent avec trois moments de l'existence : le passé, le présent et le futur. Plus précisément, Hartog définit le concept de régime d'historicité de la manière suivante : d'une part comme manière dont une société traite son passé et en traite; d'autre part comme modalité de conscience de soi d'une communauté

\footnotetext{
${ }^{17}$ Il conviendrait ici de développer une critique serrée de ce qu'on pourrait appeler le « modèle Heidegger-Moles » de l'espace où la prévalence est donnée au proche et à l'ici, où le lointain et l'ailleurs ont une valeur négative d'insécurité et où l'homme est vu comme étant isolé et nu. Cela irait trop loin ici et sera entrepris ultérieurement.

${ }^{18}$ Les “ ordres du temps" (Pomian), les “Zeitschichten " (Koselleck, 2000) et les " degrés d'historicité " (Lévi-Strauss) sont ses sources d'inspiration.
} 
humaine. Il sert à comparer des types différents d'écriture de l'histoire et de mode de rapports au temps, que Hartog appelle des " manières d'être au temps" (p. 30), des " façons d'articuler passé, présent, futur" (p. 35)

Ici, la notion de régime d'habiter vise à définir les rapports dominants, d'une civilisation - d'une " unité de survie » comme Elias (1970) appelle la société de référence -, aux lieux géographiques et à de l'espace dans leurs multiples dimensions :

I. Les spatialités, définies comme étant des manières de faire avec les agencements spatiaux et les lieux géographiques, sont devenues plus différenciées et multiples. Elles incluent les valeurs des lieux (esthétique, patrimonial, économique, résidentiel etc.) et s'incarnent dans les représentations, conceptions, images, discours d'espace (urbanisme, architecture, utopies. médias), se définissent comme étant l'imagination à l'aide de symboles de réalités qui recherchent soit la plus grande adéquation possible, soit la plus grande liberté possible avec la réalité d'espace. Elles définissent aussi, à un niveau individuel et collectif, la familiarité/étrangeté des lieux, les référents géographiques de l'identité. Les“disciplines” d'espace (Foucault, 1984 ; Harley, 1995) concernent plus particulièrement les manières dont on peut contrôler de l'espace ; les normes - entendues à la fois comme étant les règles juridiques et sociales - contribuent également à définir le régime d'habiter contemporain du point de vue de la spatialité.

II. Les pratiques des lieux et modes d'habiter - comme exposés ci-dessus - font partie du régime d'habiter. $L a$ différenciation des modes d'habiter s'entend comme étant une création de différences et de distinctions opérées par des groupes d'individus par rapport à d'autres, et ce par les systèmes cohérents de mobilité et de résidence. L'individu, c'est-à-dire le processus de développement d'un "Je " (Elias, 1970) ${ }^{20}$, inséré dans une "figuration " ou une "configuration" sociétale y acquiert des qualités spécifiques selon le régime d'habiter et les pratiques et modes d'habiter. Ses dimensions spatiales s'expriment sous formes de compétences et les référents géographiques de l'identité, souvent multiples, en font un «individu géographiquement pluriel» ou bien un habitant géographiquement pluriel et temporaire des lieux géographiques.

III. Les agencements spatiaux - définis comme étant les manières dont les lieux géographiques sont en interdépendance et se différencient les uns des autres ainsi que la différenciation spatiale des multiples phénomènes soci(ét)aux ${ }^{21}$-, les accessibilités et les qualités des lieux géographiques. La qualité des lieux se définit ici comme étant les éléments présents en un lieu en vue d'un certain usage (Brunet, 1993). Les agencements, créés dans l'action, peuvent être appréhendés dans leur organisation spatiale. Il s'agit d'espace habité en ce sens que les pratiques co-constituent les lieux géographiques et conduit à l'hypothèse de la différenciation accrue de l'espace urbain (centre-ville, mégalopole, station touristique, péri-urbain, banlieue, ville moyenne, campagne, gated community, global city pour n'en mentionner que quelques uns).

IV. Les technologies spatiales, c'est-à-dire un ensemble de machines et d'équipements sous-tendus par un discours problématisant ${ }^{22}$ constituent ainsi un "machinic complex»: «developing bundle of institutions and

\footnotetext{
${ }^{19}$ Hartog décrit trois régimes d'historicité : le passé éclairant le présent, exemplifié par l'historia magistra ; le futur éclairant le passé avec Chateaubriand et où la notion de progrès est centrale ; enfin, la période contemporaine, depuis les années 1970 , marquée par le "présentisme ", c'est-à-dire par le fait de considérer le passé en ayant en vue le présent. A sa suite, Christian Grataloup (2003) définit le " régime de géographicité " comme étant le rapport entre l'ici et l'ailleurs. Initialement, Christian Grataloup a présenté cette notion à un séminaire de l'équipe MIT portant sur la notion de " moment de lieu " (cf. Equipe MIT, 2005). Géraldine Djament (2004) rend compte d'un autre séminaire où Patrick Garcia intervenait sur l'analyse historique de l'espace.

20 “ Obgleich es zunächst den herkömmlichen Sprach- und Denkgewohnheiten zuwiderläuft, ist es viel sachgerechter, wenn man sagt, der Mensch ist ständig in Bewegung; er durchläuft nicht nur einen Prozeß, er ist ein Prozeß. Er entwickelt sich. Und wenn wir von einer Entwicklung sprechen, dann meinen wir die immanente Ordnung der kontinuierlichen Abfolge, in der jeweils eine spätere Gestalt aus früheren, in der etwa Jugend aus der Kindheit, Erwachsensein aus der Jugend ohne Unterbrechung hervorgeht. Der Mensch ist ein Prozeß" (Elias, 1970, 127). C'est traduit en français de façon suivante : «Bien que ceci heurte les habitudes linguistiques et mentales, il est pertinent de dire que l'homme est constamment en mutation. Il n'est pas soumis à un processus : il est un processus, car il se développe continûment . Et lorsque nous parlons d'évolution, nous voulons signifier l'ordre immanent à l'enchaînement continu qui fait naître une forme de celle qui la précède, comme la jeunesse naît de l'enfance et l'âge adulte de la jeunesse, et cela interruption. L'homme est un processus. » (Elias, 1991, 141). Sans pouvoir en traiter en détail, la traduction de « Bewegung » par « mutation » et non par « mouvement», et celle de « Entwicklung » par « évolution», non par « développement » pose problème en ce qu'elles sont proches du vocabulaire des sciences bio-physiques, ce qui est rejeté par Elias dans ses textes. ${ }^{21}$ Cf. la définition de Lussault (2003b) : “Assemblage spatial de réalités sociétales qui exprime l'action d'un acteur ” (p. 45). 22 “Problématisation ne veut pas dire représentation d'un objet préexistant, ni non plus création par le discours d'un objet qui n'existe pas. C'est l'ensemble des pratiques discursives ou non discursives qui fait entrer quelque chose dans le jeu du vrai et du faux et le constitue comme objet pour la pensée (que ce soit sous la forme de la réflexion morale, de la connaissance scientifique,
} 
technologies, understood as non-exclusive and diverse organisations of knowledge-discipline-perception circulating in a constantly shifting 'parliament of things' (Haraway, 1992b) : embodied subjects, machines, texts and metaphors»(Thrift, 1996: 263) ${ }^{23}$. Parmi ceux-ci, les modes de communication, technologies du déplacement, les technologies de résidence - les moyens et discours attachés à la pratique de "l'abriter", ainsi que les technologies de logistique, c'est-à-dire de la coordination spatio-temporelle des flux de biens et d'informations sont au cœur du fonctionnement mobilitaire du régime d'habiter.

V. Les situations se définissent dans/avec/par quoi les pratiques ont lieu, à la fois un moment et un site, dans leur immédiateté spatiale et temporelle (Schütz, 1932). Elles appréhendent un ensemble d'éléments permettant de situer les pratiques en question en un contexte (Fornel \& Quéré, 1999). Elles se composent de la qualité d'espace, des règles et normes sociales et des acteurs en présence. Dans le régime d'habiter actuel, elles se différencient de plus en plus, modulant le sens que les individus assignent aux lieux géographiques et obligeant à une capacité de gestion plus fine d'espace et des interactions sociales. L'un des éléments de variation des situations est constitué par les changements de lieux : passer ses vacances dans son lieu de résidence ou dans un lieu autre constitue une différence fondamentale. Un autre est la variation de l'intentionnalité : pratiquer Brighton \& Hove en tant que résident, touriste, retraité, lors d'un colloque etc. créent des situations différentes et fait varier le rapport au lieu ${ }^{24}$.

Fondamentalement, les régimes expriment un certain ordre dans lequel s'insèrent les pratiques des lieux et les individus. "Régime d'habiter" est ainsi défini comme étant le modèle dominant des manières d'être et des manières de faire avec de l'espace d'une "unité de survie", unité de référence sociétale. L'un des aspects essentiels, - il s'agit d'une hypothèse -, est que le régime d'habiter contemporain est informé par la mobilité géographique. C'est ainsi que la notion de "régime d'habiter" peut être rapprochée de l'idée de Thrift (1996) d'une "structure of feeling called mobility" des sociétés contemporaines ${ }^{25}$. Pour Thrift, le régime actuel est informé par la mobilité, car il articule trois "machinic complexes" - électricité, lumière, vitesse - d'une façon nouvelle depuis les années 1960, orientée vers la mobilité géographique.

\section{Conclusion}

Ce texte a ouvert des questionnements dans plusieurs directions : d'abord, la question de l'habiter qui n'est plus conceptualisé comme rapport à la nature ou à la Terre, mais qui devient un concept permettant de saisir théoriquement le rapport à de l'espace tel qu'il s'exprime à travers les pratiques des individus. Ensuite, que l'individu soit au centre ne signifie pas pour autant de négliger des éléments qui dépassent l'individu. Ainsi, des modèles de styles d'habiter permettent de comparer différentes manières de faire avec de l'espace et ouvrent sur la question de l'être-ensemble et la socialisation. La notion de régime d'habiter permet enfin de placer les pratiques dans un ensemble ordonnant, en leur conférant une spécificité historique. Le fil conducteur pour toutes ces analyses était la mobilité géographique : c'est à travers ce prisme que les manières de faire contemporaines avec de l'espace ont été conçues. En arrière-plan de toutes ces analyses se posait la question de la conceptualisation de l'espace : loin d'être mené à son terme, on peut retenir deux aspects. Primo, « espace» est un concept de haut niveau de synthèse qui englobe plusieurs problèmes, dont la distance, la qualité des lieux, le paysage, la localisation, l'agencement, l'orientation, l'accessibilité etc. Secundo, il s'agit toujours de l'espace habité, c'est-à-dire co-constitué par les pratiques et remplace ainsi l'idée d'un espace préexistant aux pratiques.

Le cadre théorique ainsi esquissé permet non seulement d'insérer les faits contemporains dans un système de classement, mais aussi de comprendre qu'il existe différents niveaux dans l'approche de l'habiter, et notamment dans les pratiques de mobilité : un niveau individuel et un niveau sociétal qui se soutiennent mutuellement, dialogiquement, voire "trialogiquement", car il y a trois éléments (pratique des lieux, modes d'habiter, régime

de l'analyse politique etc.) " (Foucault, 1984, p. 670) ou encore " un effort pour rendre problématiques et douteuses des évidences, des pratiques, des règles, des institutions et des habitudes qui s'étaient sédimentées depuis des décennies et des décennies " (ibid., p. 688).

${ }^{23}$ On note l'accent latourien de la théorie de l'acteur-réseau avec des « circulations » de sens et la discursivité dans un « parlement des choses» (cf. Latour, 1991; 1993).

${ }^{24}$ Cf. Stock (2001) pour un traitement approfondi de cette question.

${ }^{25}$ Thrift prend la notion de "structure of feeling " à Raymond Williams (1954) qui la définit comme étant une "culture of a period" ou " the living result of all the elements in the general organisation" (1979), cité d'après Thrift (1996: 258). Ceci est également proche de la notion d'épistémè de Michel Foucault (1966) qui définit un même paradigme, un modèle dominant de poser des questions à une époque donnée, ce que l'on pourrait appeler un régime paradigmatique. 
$\mathrm{d}^{\prime}$ habiter $)^{26}$. Les pratiques des lieux et les modes d'habiter s'insèrent obligatoirement dans un certain régime d'habiter et ce dernier est, entre autres, produit par les premiers. Toutefois, les pratiques des lieux insérées dans un régime d'habiter sont plus ou moins conformes par rapport au modèle dominant. Dans un régime d'habiter fondé sur la mobilité, tous les individus ne développent pas un mode d'habiter fondé sur la mobilité et des associations pratiques/lieux complexes. L'immobilité n'a pas perdu toute valeur: elle peut être instituée en ressource localement, mais restreint l'accès à d'autres lieux géographiques du Monde. Habiter temporairement les lieux géographiques semble en faire de ces derniers de plus en plus des «lieux de projet» pour lesquels l'ancrage et l'appropriation symbolique de la part des individus sont temporaires, toujours sujets à négociation en fonction des situations et du moment dans la vie des individus.

Cette perspective des régimes d'habiter ouvre des pistes pour des études empiriques dans différentes directions : l'étude de la constitution d'espace dans différentes situations, des façons dont la coordination spatio-temporelle s'effectue entre individus, de la composante spatiale des différentes pratiques (sous l'hypothèse de l'adéquation de plus en plus recherchée dans l'association pratiques/lieu), des styles d'habiter (plus ou moins informés par la mobilité), de la différenciation accrue de l'urbain comme «espace habité » (centre-ville, station touristique, mégalopole, péri-urbain, campagne etc.), les référents géographiques de l'identité, etc. De surcroît, dans une perspective processuelle d'un développement des sociétés - vision promue par Norbert Elias $(1970 ; 1994)$ où le passé n'est pas simplement le passé du présent, mais une étape vers un présent et un futur - il est possible d'observer différents régimes d'habiter, ayant chacun sa spécificité. Ce projet rejoint ainsi, en le complexifiant, celui d'Henri Lefebvre (1974) pour qui différentes sociétés produisaient de l'espace avec des caractéristiques spécifiques et typiques, distinguant différentes civilisations par de l'espace. Ici, ce n'est pas la production d'espace qui est au centre, mais des régimes d'habiter, centrés autour des différentes manières d'habiter des individus. Ainsi, l'Europe au Moyen-Âge, la Rome antique, Venise au $17^{\text {ème }}$ siècle, l'Europe au $19^{\text {ème }}$ siècle etc. peuvent être analysés comme étant différents régimes d'habiter dans lesquels les pratiques de mobilités ne sont pas absentes où mais la valeur sociale de la mobilité, les technologies et la qualité et l'agencement des lieux géographiques ainsi que les situations étaient autres.

Au-delà de ces études empiriques possibles, le débat sur les concepts et théories afin de contrôler, réfuter, d'amender peut être engagé.

\section{Références :}

AGLIETTA M., 1976, Régulation et crises du capitalisme, Paris, Odile Jacob (3 ${ }^{\text {ème }}$ éd. 1997)

BASSAND M., BRULHARDT M-C., HAINARD F. \& SCHULER M., 1985, Les Suisses entre la mobilité et la sédentarité. Lausanne : Presses Polytechniques Romandes

BERQUE A., 2005, “Lieux substantiels, milieu existentiel : l'espace écouménal”, in Berthoz A. \& Recht R. (dir.), Les espaces de l'homme, Paris, Odile Jacob, pp. 49-65

BERQUE A., 2002, "Habitat insoutenable. Recherche sur une histoire de la désurbanité ", L'Espace géographique, vol. 31, n 3, pp. 241-251

BERQUE A., 2000, Écoumène. Introduction à l'étude des milieux humains. Paris : Belin (coll. Mappemonde), $271 p$.

BRUNET R., 1993, “Article “Qualité””. In : BRUNET R., FERRAS R., THERY H., Les mots de la géographie. Dictionnaire critique. Paris/Montpellier : La documentation français/RECLUS, p. 409-410 (3 ${ }^{\text {ème }}$ éd.)

Bollnow O., 1999, Mensch und Raum, Stuttgart, Kohlhammer (1 ${ }^{\text {èr }}$ éd. 1963)

Cassin B. (dir.), 2004, Vocabulaire européen des philosophies, Paris, Le Seuil/Le Robert,1531p.

CERTEAU M. DE, 1990, L'invention du quotidien. 1. Arts de faire. Paris : Gallimard ( ${ }^{\text {ère éd. 1980) }}$

CHALAS Y., 2000, La ville émergente, Paris, Anthropos, 199 p.

CERIANI G., KNAFOU R. \& STOCK M., 2004, « Les compétences cachées du touriste », Sciences Humaines, vol. 145 , pp. $28-31$

DERRIDA J., 1993, Khôra, Paris, Galilée, 103 p

DJAMENT G., 2004, “Régimes d'historicité et régimes de géographicité”, Espacestemps.net (http://www.espacestemps.net/document739.html)

ELIAS N., 1997, Über die Zeit. Arbeiten zur Wissenssoziologie II. Francfort : Suhrkamp (1 ${ }^{\text {ère }}$ éd. 1984)

ELIAS N., 1994, Die Gesellschaft der Individuen. Francfort : Suhrkamp (1 ${ }^{\text {ère }}$ éd. 1987)

ELIAS N., 1991, Qu'est-ce que la sociologie?, Tour d'Aigues, Edition de 1'Aube

ELIAS N., 1970, Was ist Soziologie?, Munich, Juventus

Équipe MIT, 2005, Tourismes 2. Moments de lieux, Paris, Belin

\footnotetext{
${ }^{26}$ Après la " trialectics of space " (Soja, 1996), cette « trialogique de l'habiter » a-t-elle un sens ?
} 
Équipe MIT, 2002, Tourismes 1. Lieux communs. Paris, Belin

FORNEL M. de \& QUÉRÉ L. (dir.), 1999, La logique des situations. Nouveaux regards sur l'écologie des activités sociales, Paris, Editions de l'EHESS

FOUCAULT M., 1984, Dits et écrits II, Paris, Seuil

Fremont A., 1972, “La région : essai sur l'espace vécu ". In : Melanges offerts au professeur

A. Meynier. Saint-Brieuc : Presses Universitaires de Bretagne, pp. 663-681

FRÉMONT A., HÉRIN R., CHEVALIER J. \& RENARD J., 1984, Géographie sociale. Paris : Masson

GIDDENS A., 1994, Les conséquences de la modernité. Paris : L'Harmattan (1 ${ }^{\text {ère }}$ éd. anglaise 1990)

GOLLEDGE R.G., \& STIMSON R.J., 1997, Spatial Behavior: A Geographic Perspective. New York, Guilford Press.

HARLEY B., 1995, "Cartes, savoir et pouvoir", in : Gould P. \& Bailly A., Le pouvoir des cartes. Brian Harley et la cartographie, Paris, Economica (pp.19-51)

HARTOG F., 2003, Régimes d'historicité, Paris, Le Seuil,

HAUMONT et al., 1999, Les pavillonnaires. Paris, L'Harmattan (1 $1^{\text {ère éd. 1965) }}$

HEIDEGGER M., 2004a, “ Bauen, Wohnen, Denken ”, in : Martin Heidegger, Vorträge und Aufsätze, Stuttgart, Klett-Cotta, pp. 139-156 (1 $1^{\text {ere éd. } 1952)}$

HEIDEGGER M., 2004b, “... Dichterisch wohnet der Mensch... ”, in : Martin Heidegger, Vorträge und Aufsätze, Stuttgart, Klett-Cotta, pp. 181-198 ( $1^{\text {ère }}$ éd. 1954)

HenNig Ch., 1997, “Jenseits des Alltags. Theorien des Tourismus ”. Voyage, vol. 1, pp. 35-

53

HOYAUX F.-A. (2003) “ Les constructions des mondes de l'habitant. Éclairage pragmatique et herméneutique”, Cybergéo, n²03 (http://193.55.107.45/ehgo/hoyaux/hoyaux203.htm)

HOYAUX F.-A. (2002) “Entre construction territoriale et constitution ontologique de l'habitant. Introduction épistémologique aux apports de la phénoménologie au concept d'habiter", Cybergéo, $\mathrm{n}^{\circ} 102$ (http://193.55.107.45/ehgo/hoyaux/article.htm)

HOYAUX F.-A., 2000, Habiter la ville et la montagne. Essai de géographie phénoménologique sur les relations des habitants au lieu, à l'espace et au territoire. (Exemple de Grenoble et Chambéry), Thèse de géographie (sous la direction de Bernard Debarbieux), Université Joseph Fourier (Grenoble I)

KAUFMANN V., SCHULER M., CREVOISIER O. \& ROSSEL P., Mobilité et motilité. De l'intention à l'action, Lausanne, EPFL, Cahiers du LASUR, n 4, 2003, 76p.

KAUFMANN J.-Cl., 2001, Ego. Pour une sociologie de l'individu, Paris, Nathan

KNAFOU R., 1998, "Vers une géographie du rapport à l'Autre". In: KNAFOU R. (éd.), Les mobilités géographiques d'aujourd'hui. Paris : Ed. Belin, pp. 7-17

KNAFOU R., BRUSTON M., DEPREST F., DUHAMEL PH., GAY J.-CH. \& SACAREAU I., 1997, “Une approche géographique du tourisme ". L’Espace géographique, vol. 26, nº3, pp. 193-204

KOSELLECK R., 2000, Zeitschichten. Studien zur Historik, Francfort, Suhrkamp

LAHIRE B., 2004, La culture des individus. Dissonances culturelles et distinction de soi, Paris, La Découverte

LAHIRE B., 1998, L'homme pluriel. Les ressorts de l'action, Paris, Nathan

LAtour B., 1993, La clef de Berlin et autres leçons d'un amateur de sciences. Paris, Le Seuil

LAtour B., 1991, Nous n'avons jamais été modernes. Essai d'anthropologie symétrique.

Paris : La Découverte

LAZZAROTTI O., 2001, La raison de l'habiter. Patrimoine et tourisme, Dossier HDR, Université de Paris 7Denis Diderot

LEFEBVRE H., 1999, La production de l'espace, Paris, Anthropos, (1 ère éd. 1974)

LÉVY J., 2004, Les échelles de l'habiter, Rapport de recherche

LÉVY J., 2003, "Capital spatial", in : LuSSAULT M. \& LEVY J. (dir.), Dictionnaire de la géographie et de l'espace des sociétés, Paris, Belin

LÉVY J., 1999, Le tournant géographique. Paris : Belin (coll. Mappemonde)

LÉVY J., 1994, L'espace légitime. Sur la dimension géographique de la fonction politique. Paris : Presses de la Fondation Nationale des Sciences Politiques

LÉVY J. \& HAEGEL F., 1997, "Urbanités, identité spatiale et représentation de la société », in Calenge C., Lussault M. \& Pagand B. (dir), Figures de l'urbain; des villes, des banlieues et de leurs représentations, Publications de la maison des sciences de la ville, p35-95.

LÉVY J. \& LUSSAULT M., 2003, “ Habiter ”, in : LUSSAULT M. \& LEVY J. (dir.), Dictionnaire de la géographie et de l'espace des sociétés, Paris, Belin 
LUSSAULT M., 2003a, “Spatialité ”, in : LuSSAult M. \& LeVY J. (dir.), Dictionnaire de la géographie et de l'espace des sociétés, Paris, Belin

LUSSAULT M., 2003b, “Agencement spatial”, in: LuSSAult M. \& LeVY J. (dir.), Dictionnaire de la géographie et de l'espace des sociétés, Paris, Belin

LUSSAUlT M., 2000, “Action(s)! ”. In : LuSSAult M., LeVy J. (dir.), Logiques de l'espace, esprit des lieux. Géographies à Cerisy. Paris : Belin, pp. 11-36

MacCANNELL, 2004, « Tourist Agency », Tourist Studies, vol. 1, n¹, pp. 23-37

MAYOL P., 1990, “Habiter”, in : Michel de Certeau, Luce Giard \& Pierre Mayol, L'invention du quotidien 2.

Habiter, cuisiner, Paris, Gallimard

MERLEAU-PONTY M., 1945, La phénoménologie de la perception, Paris, Gallimard

MOLES A., 1995, “Vers une psycho-géographie”, In : BAILly A., FERRAS R. \& PUMAIN D. (éd.), Encyclopédie de Géographie. Paris : Economica, pagination?

OLLIVRO J., 2000, L’homme à toutes vitesses. De la lenteur homogène à la rapidité différenciée. Rennes : Presses Universitaires de Rennes

PIOLLE X., 1991, “Proximité géographique et lien social, de nouvelles formes de territorialité ?". L'Espace géographique, vol. 20, $\mathrm{n}^{\circ}$ 4, pp. 349-358

Piolle X., 1990, "Mobilité, identités, territoires". Revue de Géographie de Lyon, vol. 65, $\mathrm{n}^{\circ 3}$, pp. 149-154

POPPER K, 1998, La connaissance objective, Paris, Champs Flammarion, 578p. (1 ${ }^{\text {ère }}$ éd. 1979)

RADKOWSKI G.-H. de, 2002, Anthropologie de l'habiter. Vers le nomadisme, Paris, PUF (1 ${ }^{\text {ère }}$ éd. 1962-1968)

RELPH E., 1971, "An Inquiry onto the Relations between Phenomenology and Geography".

Canadian Geographer, vol. 14, n³, pp. 193-201

RÉMY J., 1996, "Mobilités et ancrages : vers une autre définition de la ville", In : HIRSCHHORN M., BeRTHELOT J.-M. (dir.), Mobilités et ancrages. Vers un nouveau mode de spatialisation ? Paris : L'Harmattan, pp. $135-153$

ROCHE D., 2000, La mobilité à Paris. Paris, Fayard

SACAREAU I. \& STOCK M., 2003, "Qu'est-ce que le tourisme?”, in: Stock M. (coord.), Le tourisme. Acteurs, lieux et enjeux. Paris, Belin

SCALAB, 2004, Échelles de l'habiter. Contrat de recherche avec le PUCA, Paris.

SeAmon D., Mugerauer R., 1989, Dwelling, place and environment. Towards a Phenomenology of Person and World. Dordrecht: Martinus Nijhoff

SCHULZE G., 1997, Die Erlebnisgesellschaft. Kultursoziologie der Gegenwart, Munich : Campe (1 ${ }^{\text {ère }}$ éd. 1994) SOJA E., 2001, Postmetropolis. Critical Studies of Cities and Regions. Londres : Blackwell

SPODE H., 2004, « Fordism, mass tourism and the Third Reich: The "strength through joy" seaside resort as an index fossil », Journal of Social History, 38 (1), pp.127-155

STOCK M., 2005, "Les sociétés à individus mobiles: un habiter poly-topique ? L'exemple des pratiques touristiques », Espacestemps.net, Textuel, 25.05.2005 (http://www.espacestemps.net/document1353.html)

STOCK M., 2004, “L'habiter comme pratique des lieux”, Espacestemps.net, Textuel, 18.12.2004 (http://www.espacestemps.net/document1138.html)

STOCK M., 2001, Mobilités géographiques et pratiques des lieux. Étude théorico-empirique à travers deux lieux touristiques anciennement constitués: Brighton \& Hove (Royaume-Uni) et Garmisch-Partenkirchen (Allemagne), thèse de géographie, Université de Paris 7 - Denis Diderot, 663p.

STOCK M. \& DUHAMEL Ph., 2005, “A practice-based approach to the conceptualisation of geographical mobility ”. BELGEO - Revue belge de géographie, $\mathrm{n}^{\circ} 1-2$, pp. 59-68

TARRIUS A., 1992, Les fourmis d'Europe : migrants riches, migrants pauvres et nouvelles villes internationales, Paris, L'Harmattan

THRIFT N., 1996, Spatial Formations, Londres, Sage

TUAN Y.-F., 1971, "Geography, Phenomenology and the Study of Human Nature".

Canadian Geographer, vol. 14, n², pp. 193-201

URRY J., 1990, The Tourist Gaze, Londres, Sage

VIARD J., 1994, La société d'archipel ou les territoires du village global. Paris : Éditions de l'Aube (coll. Monde en cours)

WERLEN Benno, 1997, Sozialgeographie alltäglicher Regionalisierung, tome 2 : Globalisierung,

Region und Regionalisierung. Stuttgart, Steiner, (2 ${ }^{\text {ème }}$ éd. 1999)

WERLEN Benno, 1996, “Geographie globalisierter Lebenswelten ”, Österreichische Zeitschrift für Soziologie, vol. $21, \mathrm{n}^{\circ} 2$, pp. $97-128$ 
WERLEN Benno, 1995, Sozialgeographie alltäglicher Regionalisierung, tome 1 : Zur Ontologie von Gesellschaft und Raum. Stuttgart, Steiner, (2 ${ }^{\text {ème }}$ éd. 1999)

WERLEN Benno, 1987, Gesellschaft, Handlung, Raum. Stuttgart, Steiner, (3 ${ }^{\text {ème }}$ éd. 1999, ed. anglaise Society, Action and Space. An Alternative Human Geography, Londres, Routledge, 1993) 